\title{
Tolerante Rassist*innen: Kämpfe um Anerkennung in der deutschen Stadt
}

Rezension zu Peter Bescherer / Anne Burkhardt / Robert Feustel / Gisela Mackenroth / Luzia Sievi (2021): Urbane Konflikte und die Krise der Demokratie. Stadtentwicklung, Rechtsruck und Soziale Bewegungen. Münster: Westfälisches Dampfboot.

Tino Buchholz

Abb. 1 Titel des Buches (Quelle: Westfälisches Dampfboot)

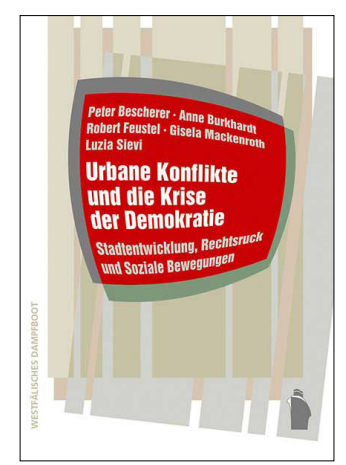

Der Band Urbane Konflikte und die Krise der Demokratie umfasst einen zeitgemäßen Titel in der Reihe „Raumproduktionen“ des Dampfbootverlags. Er widmet sich der Problematik rechter Stadtpolitik in Deutschland und wird in seiner Aktualität nur durch den 2012 erschienen Band 14 dieser Reihe (Bürk 2012) übertroffen. Bei dem vorliegenden Sammelband handelt es sich um eine Veröffentlichung des Forschungsprojekts Populismus und Demokratie im Stadtraum (PODESTA), in dem zwei soziologische Forschungsteams aus Jena und Tübingen rechten Bewegungen in der Raumproduktion in Leipzig und Stuttgart nachgehen. Auch die politische Rechte hat ein Bild von der guten Stadt. Insofern ist die Tatsache, dass rechte Bewegungen Identitätspolitik für sich reklamieren, kein Zufall. Sozialräumliche Anerkennung - oder Missachtung - als für erstrebenswert erachtete Werte sozialen Lebens sind fester Bestandteil normativer Ordnung. Die Frage ist also eher, worin das Soziale sozialer Bewegungen besteht oder anders formuliert: Wer mobilisiert welche Werte, wann, wo, wie, warum und mit welchem Erfolg? Welche Formen sozialer Inklusion und räumlicher Repräsentation lassen sich beobachten? Wo verlaufen Grenzen? Diese Fragen lassen sich von links wie rechts beantworten. Mit meiner anerkennungstheoretischen Lesebrille habe ich mich folglich an die stadtpolitische Lektüre rechter Identitätsformation gemacht.

In ihrer Einleitung warnen Peter Bescherer und Gisela Mackenroth (2021) sogleich vor einer vereinfachenden Gegenüberstellung von Stadt und Land: „Rechtspopulismus, Rassismus, Antifeminismus und Demokratiefeindlichkeit gibt es auch in urbanen Räumen“ (ebd.: 11). Dieser Prämisse folgend fokussieren die Autor*innen spezifisch städtische Ursachen rechter Stadtpolitik sowie Fragen, wie populistische Lücken entstehen, wie sie sich von rechts besetzen lassen und welche Rolle demokratische Prozeduren für inklusive Stadtpolitik spielen. Wenn der Stadt-Land-Unterschied so nicht haltbar ist, drängt sich die Frage auf, wie der begrenzte Rahmen (Sozialisation und Bildung) zu erklären ist, in dem ein identitär verengtes „Wir“ die Angst vor „Anderen“ bedeutet und sich populistisch instrumentalisieren lässt (vgl. Belina 2021). Was konstituiert das Gemeinsame? Wo haben solidarisch 
geteilte Werte in Gemeinschaft und Gesellschaft ihre Grenzen - im Denken und Handeln? Was bedeutet das für tolerante Rassist*innen und die „reinrassige“ Identitätsformation rechter Bewegungen? Diesen normativen Fragen sozialräumlicher Ordnung - und scale - spüren die Autor*innen mithilfe der dritten Generation der Frankfurter Schule nach (vgl. Buchholz 2021).

Das Grundgerüst der Argumentation liefert Helmut Dubiels (1985) Gespenst des Populismus. Darin bemüht er den Zwiespalt zwischen rationalen Imperativen und affektiver Interessenvertretung und fokussiert die (historische) Tatsache, „daß das Proletariat oft anders gehandelt hat, als es ihm sein objektives Klasseninteresse geboten hätte“(ebd.: 647). Im Kern geht es Dubiel um nichts weniger als um eine politische Theorie sozialen Wandels und ein Verständnis politischer Subjektivität, um ,jene schwer greifbaren, dem Alltagsbewusstsein eher latent präsenten Glückserwartungen, Gerechtigkeitsansprüche, Bedürfnisse nach sozialer Anerkennung und kultureller Identität“ (ebd.: 648), die durch politische, kulturelle Eliten vordefiniert und durch das Volk als Kränkung empfunden werden.

Im Zentrum der vorliegenden Studie steht die Volk-Elite-Dichotomie, die Bedrohung der deutschen Urstadt - von innen und außen - sowie die Abwehr gegen Wissenschaft, Medien und Volksparteien, die im Widerspruch zum gesunden Menschenverstand stünden. Die Bedrohung von innen wird dem Staat und seinen staatstragenden Institutionen zugeschrieben, die sich als unfähig oder unwillig erwiesen, die deutsche Stadt zu schützen. Die angebliche Bedrohung durch Migration übersteigt jene der sogenannten Volksverräter*innen von innen, wenn äußerlichen Feinden unterstellt wird, die Homogenität des Volkskörpers anzutasten. Folglich sprechen Anne Burkhardt und Robert Feustel (2021) in Kapitel 2 von Machtübernahme, Machtübergabe und widerständiger Rückeroberung der deutschen Stadt: mit Blick auf städtische Sicherheit, urbane Mobilität und sozialen Wohnraum. Während städtische Sicherheit das bevorzugte Schlachtfeld rechter Narrative bedeutet, spielt die „Sehnsucht nach etwas Verlorenem“ (ebd.: 51) für die automobile Verkehrspolitik eine sekundäre Rolle. Mit Blick auf Stuttgart geht es um Gegenwart und Zukunft der deutschen Automobilindustrie sowie darum, „Wut auf die Politik und Angst vor dem Verlust von Eigentum, Arbeitsplätzen und Privilegien in der Bevölkerung [zu] schüren“ (ebd.). Die Bedrohung von außen wird an dieser Stelle nicht von Migration markiert, sondern von der EU und der asiatischen Autoindustrie. Bei der Wohnraumdebatte ist Migration jedoch wieder das vermeintlich zentrale Übel, ohne das der deutsche Wohnungsmarkt keine Probleme hätte. „Die von rechts skizzierten Wege zu mehr Wohnraum und günstigeren Mieten [...] oszillieren zwischen marktradikal und sozial-national“ (ebd.: 52).

Peter Bescherer (2021a) vertieft diese Argumentation in Kapitel 3 und fragt nach Problemwahrnehmung, Ursachensuche und Gegenstrategien in Leipzig. Dievon ihm interviewten Expert*innen aus Stadtrat, Gewerkschaften und Sozialer Arbeit zeigen sich eher ratlos und können keine stadtpolitische Expertise der AfD erkennen: „Rechtspopulistische Ressentiments gegen Migrant*innen und Eliten erklären sie vor dem Hintergrund städtischer Probleme, insbesondere der ungleichen räumlichen Entwicklung und der damit einhergehenden Herausbildung erzwungener Nachbarschaften." (Ebd.: 68) So verweist Bescherer zu Recht auf über- und tiefer gelagerte 
scales der lokalen Verarbeitung doppelter Transformation, in der sich betagte Leipziger*innen gesellschaftlich und biografisch zwischen Staatssozialismus und globalisiertem Kapitalismus aufgerieben fühlen.

„Die Ratlosigkeit der Zuständigen II“ beschäftigt auch Robert Feustel (2021) in Kapitel 4, denn auch seine Ursachenforschung kann keine explizit stadtpolitische Dimension der sozialräumlichen Erklärung rechter Narrative ausmachen. Was noch nicht ist, kann jedoch als „Wette auf eine möglicherweise düstere Zukunft“ (ebd.: 92) städtischer Politik verstanden werden - so Feustel mit Blick auf populistische Lücken, selektive Beteiligung und mangelnde Inklusion. Während die Autor*innen des Buches zunächst die explizit stadtpolitische Dimension rechter Narrative fokussieren, führt sie die Ratlosigkeit der institutionell Verantwortlichen zur Modellierung ihres Forschungsdesigns. Für Feustel liegt es an dieser Stelle nahe, den Fokus auf das Alltagsleben zu verschieben, um Affekte und Emotionen im Alltag besser zu verstehen und „eine in Teilen ,entortete“ Politik auf ihre Bezüge zum Sozialraum hin empirisch zu untersuchen“ (ebd.: 96).

Die zweite Hälfte des Bandes widmet sich daher den Mikrokonflikten der Lebenswelt und ihrer Nähe zu rechten Narrativen. Den Anfang macht eine Garagengemeinschaft im Leipziger Osten, die einem Gymnasium weichen soll (Kapitel 5). Obwohl die Zustimmung für den Schulneubau im Stadtteil groß ist, vermag es die AfD, die populistische Lücke durch selektive Beteiligung am Planungsprozess zu füllen. Was zunächst nach einem intergenerationellen Konflikt aussieht, entpuppt sich als Dilemma egalitärer Teilhabe in der Stadtplanung.

„Die Pächter erzählten uns ungefragt, dass man auf Landes- und vor allem auf Bundesebene die Politik der AfD äußerst kritisch betrachten müsse. Lokal sei sie aber die einzige Partei, die als ihre Fürsprecherin auftritt. [...] Die Annäherung an die AfD gleicht in diesem Fall eher dem Versuch einer Instrumentalisierung der Instrumentalisierer als einer überzeugten Hinwendung zur rechtspopulistischen Programmatik.“ (Bescherer/Feustel 2021: 119)

Auch in den Stuttgarter Gelbwestenprotesten (Kapitel 6) spielt die AfD ihre Rolle, jedoch nicht die entscheidende. Die überparteilichen Proteste lassen zwar eine klare Abgrenzung von rechten Narrativen vermissen, können aber dennoch als dankbares Thema für rechte Akteur*innen gelten. So eignen sie sich, um wissenschaftliche Uneinigkeit und demokratische Streitkultur als links-grünen Populismus umzudeuten und zurück zu projizieren. Die dieselverbundene Geschichte des Wirtschafts- und Sozialraums Stuttgart bringt es mit sich, dass die Stadt der Verkehrswende zumindest gespalten gegenübersteht; was die Debatte um Demokratiedefizite und Fremdbestimmung befeuert, und den automobilen, traditionalistisch männlichen Lebensstil infrage stellt. Während dieses Kapitel männliche Verlustängste sehr deutlich nachzeichnet, trägt die Parallele zur Gelbwestenbewegung in Frankreich allerdings nicht. Die gut bezahlten Facharbeiter*innen der Stuttgarter Autoindustrie verbinden mit der gelben Weste zwar ein Symbol für Protest, nicht aber ihren Status.

Gisela Mackenroth (2021) fokussiert in Kapitel 7 das wohnungspolitische Konfliktpotenzial in der für Mieter*innen teuersten Stadt Deutschlands. Ihr 
Kapitel dokumentiert keinen zählbaren Widerstand, sondern eine eindringliche Beschreibung des Politischen alltäglicher Nachbarschaft im Nordosten Stuttgarts. Der Stadtteil Hallschlag ist eine migrantisch geprägte Arbeiter*innensiedlung in unmittelbarer Nähe zur Daimlerproduktion, wo der Fahrzeugbauer ebenfalls Wohnungen unterhielt, später aber verkaufte. Die lokal-staatlich fazilitierte, soziale Durchmischung zugunsten einkommensstärkerer Haushalte (state-led gentrification) ging nicht spurlos an den Bewohner*innen des Viertels vorüber, wie Frau Otto, die Protagonistin des Kapitels zu berichten weiß. Im Unterschied zu den anonymisierten Interviews der Garagensiedlung in Leipzig begibt sich Mackenroth auf ethnographische Spurensuche und gibt den Leser*innen Namen - das macht Lust auf mehr.

Obwohl deutlich wird, dass Frau Otto einst einvernehmlich mit ihren türkischen Nachbar*innen zusammen wohnte, werden Ursprung und Ausprägung ihrer rassistischen Ressentiments nur schemenhaft skizziert. Als Gründe für den Aufstieg der AfD benennt die Autorin zwar ökonomische Härten, eine geringe Wahlbeteiligung sowie die Tatsache, dass 40 Prozent der Bewohner*innen des Stadtteils überhaupt keinen deutschen Pass besitzen und somit auch nicht wahlberechtigt sind. An dieser Stelle hätte sich der*die geneigte Leser*in jedoch ein wahrhaftiges Tiefeninterview gewünscht, das zwischen vormaliger Anerkennung leistungsbezogener Migration und Toleranz unterscheidet und diese analytisch von der aktuellen Missachtung nichtdeutscher Nachbar*innen abgrenzt. Entgegen einer dichteren Beschreibung ihrer Person, Generation und Lebenswelt - nebst der eventuell abweichenden Wahrnehmung ihrer Söhne (die gemeinsam mit ihren türkischen Nachbar*innen aufwuchsen und spielten) führt Mackenroth Entfremdung als analytische Kategorie der Studie ein, was ich als überaus fruchtbar empfinde.

Auch Peter Bescherer (2021b) bemüht die Entfremdung der Bewohner*innen als zentrales Argument seiner Fallstudie in Leipzig (Kapitel 8), während ein imaginierter Gegner den Blick auf Vonovia und eine adäquate Kritik der wohnungspolitischen Problemlage verstellt. Anstatt den größten privaten Akteur auf dem deutschen Wohnungsmarkt zu fokussieren, geraten nichtdeutsche Nachbar*innen in Kritik für überzogene Nebenkostenabrechnungen, erhöhte Fluktuation und gesunkene Lebensqualität in der Nachbarschaft. Bescherers Ethnographie der Ethnographie liefert eine dichte Beschreibung aktivistischer Transformationsstrategien des Leipziger Netzwerks „Stadt für Alle“, zu dem der Autor selbst gehört. Den Aktivist*innen geht es um nichts weniger als den Aufbau einer mietenpolitischen Bewegung und den Ausbruch aus der eigenen Blase: Ein delikates, aber machbares Unterfangen, wie das Beispiel zeigt - mit klarer Kante gegen tolerante Rassist*innen und Lerneffekten für beide Seiten. Bescherers Beschreibung einer unvollständig vollzogenen, ökonomischen Entfremdung könnte an dieser Stelle Anlass zur Hoffnung für de-kommodifizierte Perspektiven auf Wohnraum geben, wäre hier nicht noch politische Entfremdung zu konstatieren, sodass sich völkische Motive mit demokratischen Prozeduren vermischen und rechte Erzählungen von Überfremdung befeuern. Bescherer macht durchaus deutlich, dass die vorliegende Studie vorerst keine Typologie der entfremdeten, rassifizierten Wohnungsfrage leistet. Diese steht für die Stadtforschung noch aus. 
Im Schlusskapitel lassen die Autor*innen Entfremdung und scale der sozialräumlichen Lebenswelt als Leitlinien der Analyse auf ihrem Weg liegen. Stattdessen richten Feustel und Sievi (2021) den Blick auf die Demokratisierung der Stadtpolitik, auf das transformatorische Potenzial städtischer Bewegungen sowie auf andauernde Kämpfe um Anerkennung (vgl. Honneth 1994), die sie jedoch nicht so nennen. Im Ergebnis hätte eine Berücksichtigung zeitgenössischer Protagonist*innen der Frankfurter Schule der vorliegenden Studie sicherlich gutgetan, um Anerkennung, Missachtung und Toleranz analytisch klar voneinander abzugrenzen (vgl. Forst 2003) und um vorhandene rassistische Ressentiments mithilfe politischer Subjektivität durchzudeklinieren - historisch, geographisch, legal, sozial und emotional. Der Anfang ist jedoch gemacht. So ließe sich das modellierte Forschungsdesign auf andere deutsche Städte übertragen sowie moralgeographisch und sozialanthropologisch zuspitzen. Zeitgleich leistet der Sammelband eine empirisch reichhaltige, analytische Suchbewegung, die überaus gelungen ist.

\section{Autor_innen}

Tino Buchholz ist Stadtforscher und Filmemacher. Seine Forschung fokussiert kritische soziale Theorie und räumliche Praxis in der Stadt- und Wohnungspolitik.

tino.buchholz@si.uni-stuttgart.de

\section{Literatur}

Belina, Bernd (2021): „Provinzialität“ bei Adorno. In: Geographische Zeitschrift 109/2-3, 105-125.

Bescherer, Peter / Burkhardt, Anne / Feustel, Robert / Mackenroth, Gisela / Sievi, Luzia (Hg.) (2021): Urbane Konflikte und die Krise der Demokratie. Stadtentwicklung, Rechtsruck und Soziale Bewegungen. Münster: Westfälisches Dampfboot.

Bescherer, Peter (2021a): Die Ratlosigkeit der Zuständigen I. Beim scale jumping verloren gegangen? In: Peter Bescherer / Anne Burkhardt / Robert Feustel / Gisela Mackenroth / Luzia Sievi (Hg.), Urbane Konflikte und die Krise der Demokratie. Stadtentwicklung, Rechtsruck und Soziale Bewegungen. Münster: Westfälisches Dampfboot, 59-77.

Bescherer, Peter (2021b): Solidarität durch Mieterkämpfe? Nachbarschaftsorganizing im Leipziger Nordosten. In: Peter Bescherer / Anne Burkhardt / Robert Feustel / Gisela Mackenroth / Luzia Sievi (Hg.), Urbane Konflikte und die Krise der Demokratie. Stadtentwicklung, Rechtsruck und Soziale Bewegungen. Münster: Westfälisches Dampfboot, 175-205.

Bescherer, Peter / Feustel, Robert (2021): Von der Parklücke zur populistischen Lücke? Der Streit um einen Garagenhof in Leipzig. In: Peter Bescherer / Anne Burkhardt / Robert Feustel / Gisela Mackenroth / Luzia Sievi (Hg.), Urbane Konflikte und die Krise der Demokratie. Stadtentwicklung, Rechtsruck und Soziale Bewegungen. Münster: Westfälisches Dampfboot, 98-121.

Bescherer, Peter / Mackenroth, Gisela (2021): Vom Recht auf Stadt zur Stadt von rechts und zurück. In: Peter Bescherer / Anne Burkhardt / Robert Feustel / Gisela Mackenroth / Luzia Sievi (Hg.), Urbane Konflikte und die Krise der Demokratie. Stadtentwicklung, Rechtsruck und Soziale Bewegungen. Münster: Westfälisches Dampfboot, 10-35.

Buchholz, Tino (2021): New directions in Frankfurt critical theory for critical urban theory. In: Anthony Orum / Javier Ruiz-Tagle / Serena Vicari (Hg.), Companion to WileyBlackwell encyclopedia of urban and regional studies. Oxford: Wiley Blackwell, 155-175.

Burkhardt, Anne / Feustel, Robert (2021): Die Stadt als Projektionsfläche. Antiurbane Narrative von rechts. In: Peter Bescherer / Anne Burkhardt / Robert Feustel / Gisela Mackenroth / Luzia Sievi (Hg.), Urbane Konflikte und die Krise der Demokratie. Stadtentwicklung, Rechtsruck und Soziale Bewegungen. Münster: Westfälisches Dampfboot, 36-58. 
Bürk, Thomas (2012): Gefahrenzonen, Angstraum, Feindesland? Stadtkulturelle Erkundungen zu Fremdenfeindlichkeit und Rechtsradikalismus in ostdeutschen Kleinstädten. Münster: Westfälisches Dampfboot.

Dubiel, Helmut (1985): Das Gespenst des Populismus. In: Merkur 39/8, 639-651.

Feustel, Robert (2021): Die Ratlosigkeit der Zuständigen II. Zwischen Skepsis und Sorge. In: Peter Bescherer / Anne Burkhardt / Robert Feustel / Gisela Mackenroth / Luzia Sievi (Hg.), Urbane Konflikte und die Krise der Demokratie. Stadtentwicklung, Rechtsruck und Soziale Bewegungen. Münster: Westfälisches Dampfboot, 78-97.

Feustel, Robert / Sievi, Luzia (2021) Für eine Demokratisierung der Städte. In: Peter Bescherer / Anne Burkhardt / Robert Feustel / Gisela Mackenroth / Luzia Sievi (Hg.), Urbane Konflikte und die Krise der Demokratie. Stadtentwicklung, Rechtsruck und Soziale Bewegungen. Münster: Westfälisches Dampfboot, 206-225.

Forst, Rainer (2003): Toleranz im Konflikt. Berlin: Suhrkamp.

Honneth, Axel (1994): Kampf um Anerkennung. Berlin: Suhrkamp.

Mackenroth, Gisela (2021): Wie das Fahrradhäuschen die Wäscheleine verdrängt. Alltag und Ressentiment in einem Stuttgarter Quartier „mit besonderem Entwicklunsgbedarf“. In: Peter Bescherer / Anne Burkhardt / Robert Feustel / Gisela Mackenroth / Luzia Sievi (Hg.), Urbane Konflikte und die Krise der Demokratie. Stadtentwicklung, Rechtsruck und Soziale Bewegungen. Münster: Westfälisches Dampfboot, 153-174. 